\title{
Differentiated methodology of coordinating abilities upbringing in children with infantile cerebral paralysis
}

\author{
Artem Poteshkin* and Irina Talamova \\ Siberian State University of Physical Educations and Sport, 644009, Omsk, Russia
}

\begin{abstract}
In the article the authors give the example of own created methodology of coordinating abilities estimation and upbringing in children with spastic diplegia and hemiparetic forms of infantile cerebral paralysis at the age of 11-12 of the Ist level of functional abilities according to Gross Motor Function Classification Systems. The peculiarity of the methodology is its universal character: the created methodology of estimation helps to estimate within a short time period and in terms of free space deficiency estimate coordinating abilities development. According to the development results estimation of a girl with a hemiparetic form of infantile cerebral paralysis we see the best indices of coordinating abilities development in comparison with the girls with spastic diplegia and the boys of both forms. For coordinating abilities upbringing the authors offer the methodology. It includes two blocks: the $1^{\text {st }}$ blockclassical physical exercises; the second block- exercises at stable platform. The authors offer the algorithm of coordinating abilities estimation and upbringing in children with spastic diplegia and hemiparetic forms of infantile cerebral paralysis at the age of 11-12.
\end{abstract}

\section{Introduction}

The importance of coordinating abilities upbringing in children with infantile cerebral paralysis (ICP) is obvious, as social adaptation, educational abilities and needs of such children depend on the level of coordinating abilities [1]. At the same time, ICP diagnosis influences the level of coordinating abilities development. The degree of the disease developed conditions the ability of a child with ICP differentially fulfill motor actions, balance on the support, estimate spatial, time and dynamic characteristics of a movements [2].

In prognostic meaning such forms of ICP as spastic diplegia and hemiparetic form are more favorable for motor and social restrictions overcoming. For different forms of ICP there are different methodologies of coordinating abilities upbringing using different pedagogical and computer technologies [3].

\section{Materials and methods}

We consider the technologies, which help to get information from the systems of an organism on-line. Such technologies include audiovisual feedback (AVFB) [4-6]. Classical pedagogical (standard methodologies of coordinating abilities upbringing by means of physical exercises) and computer technologies (received signals about the position of pressure center on the surface with further processor treatment and results demonstration for the preserved analyzers of children with ICP) combination improves the process of coordinating abilities upbringing [7,8].

We tried to create the methodology of coordinating abilities estimation [9] and upbringing among children with spastic diplegia and hemiparetic form of ICP at the age of 11-12 of the Ist level of functional abilities according to GMFCS. We tried to integrate the lessons at force plate "Stabilan-01-2" (Close Joint-stock Company Special Design Bureau "Rhythm"). The methodology includes two parts: 1) Coordinating abilities estimation; 2) Coordinating abilities upbringing.

Coordinating abilities estimation among children with spastic diplegia and hemiparetic forms of infantile cerebral paralysis included 32 children at the age of 11-12 of the Ist level of functional abilities according to Gross Motor Function Classification Systems (GMFCS) [10]. According to the development results estimation of a girl with a hemiparetic form of infantile cerebral paralysis we see the best indices of coordinating abilities development in comparison with the girls with spastic diplegia and the boys of both forms [11]. It can be explained by more mature development of girls' cerebrum structures. They play great role in coordinating abilities demonstration and also by the fact that hemiparetic form of ICP is more favorable in terms of ICP consequences overcoming. It is proved by the research works of F.A. Yunusov and L.V. Shapkova. They consider this form of ICP more favorable for all kinds of rehabilitation [3].

\footnotetext{
* Corresponding author: artempoteshkin91@mail.ru
} 
For coordinating abilities upbringing we used physical exercises, selected by Kalmykov D.A. for children with ICP [12]. Originality of our approach is in the fact that we added the exercises at force plate ("Stabilan-01-2", computer program "StabMed" version 2.08) to this list.

\section{Results and discussion}

For more detailed methodology understanding we created the algorithm. It helps to present the whole process in a form of block-schemes (Fig. 1).

\section{Filling in the informed agreement by legal representative (if the child is not 14)}

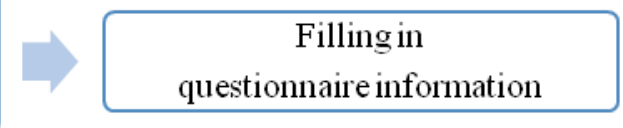

Coordinating abilities of the upper extremities estimation in children with ICP with spastic diplegia and hemiparetic forms

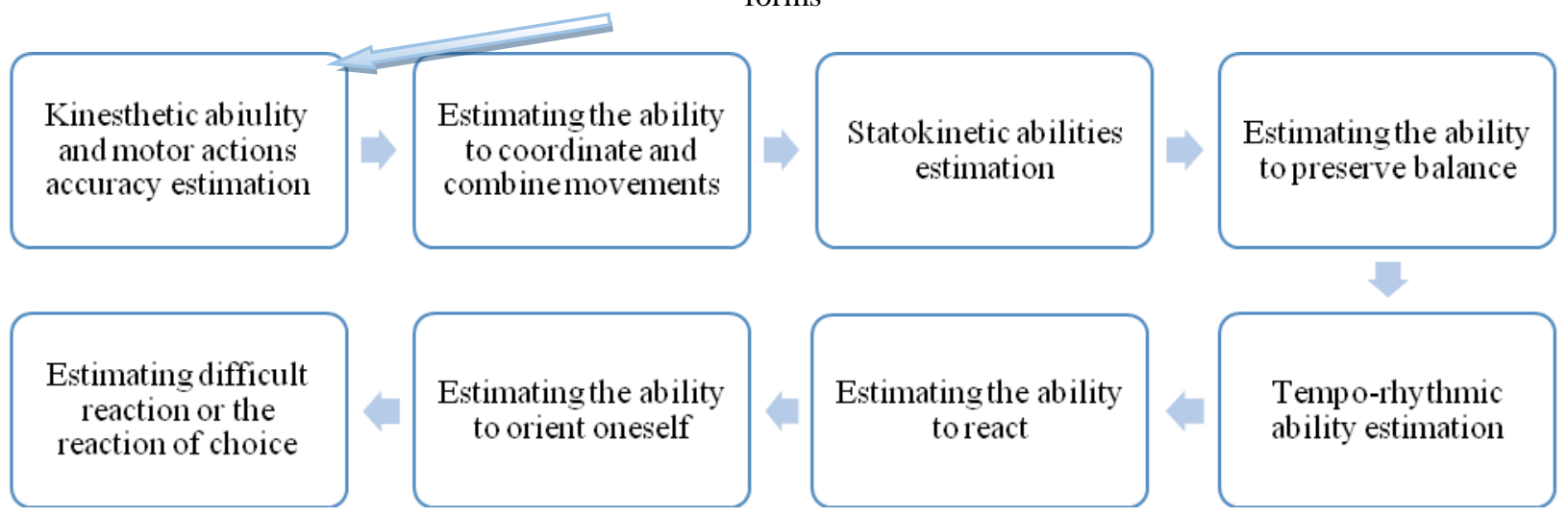

Coordinating abilities of the lower extremities estimation in children with ICP with spastic diplegia and hemiparetic forms

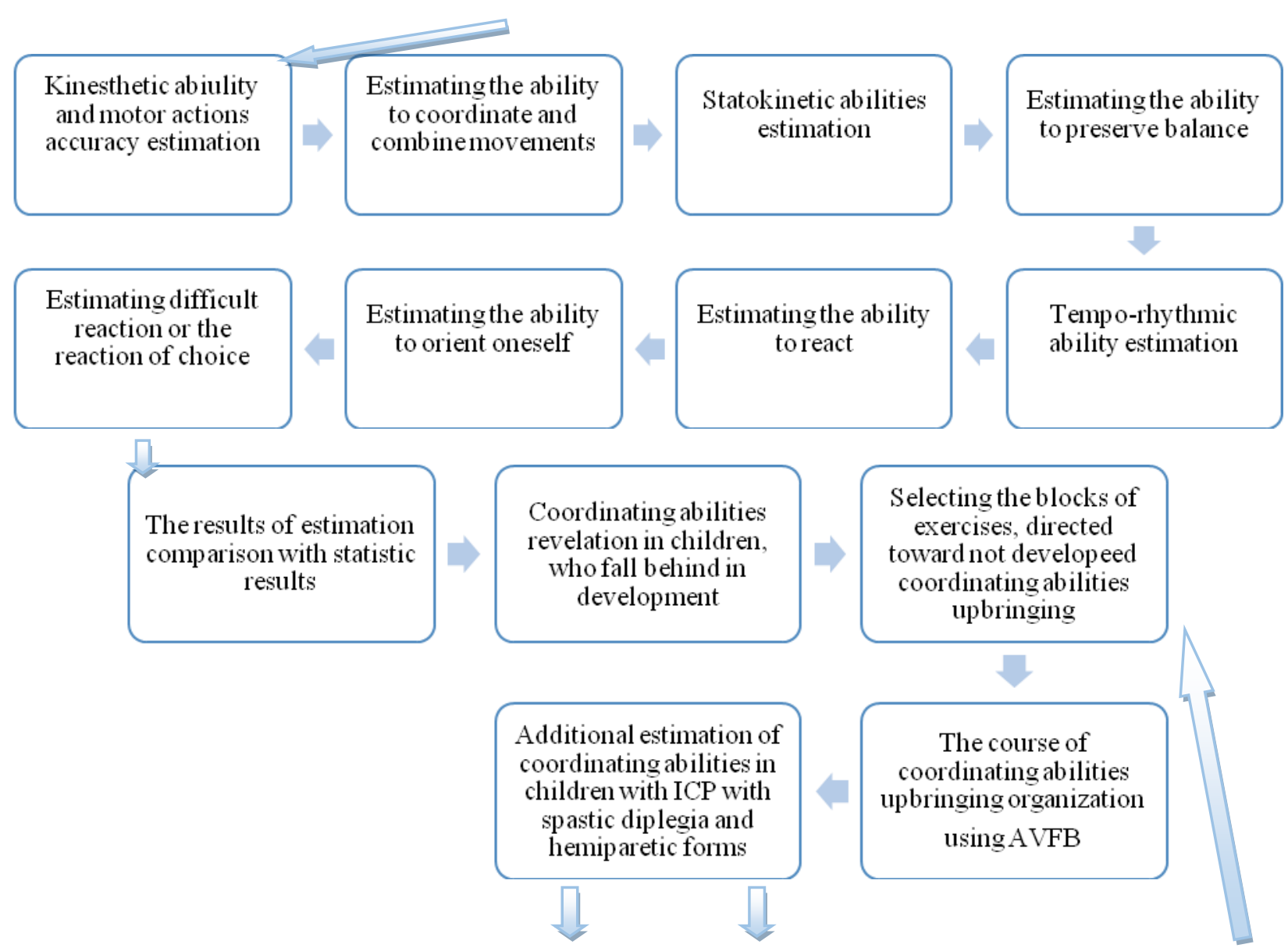


The indices increase of not developed coordinating abilities
No changes or decrease of indices of coordinating abilities development

Fig. 1. Algorithm of coordinating abilities estimation and upbringing in children with ICP with spastic diplegia and hemiparetic forms of the Ist level of functional abilities according to GMFCS

After coordinating abilities revelation, among those who fall behind in development, we offer the methodology of upbringing, presented in a form of blocks of exercises. The structure of a single lesson according to this methodology is the following:

- introductory part (1-2 minutes);

- preparatory part (5-6 minutes);

- the main part (20 minutes);

- final part (2 minutes).

General time of the lesson: 30 minutes.

During the introductory part we explained the objectives of the lesson, gave instructions to children concerning safety technique.

During the preparatory part children fulfills general developing exercises, which prepare the organism of a child with ICP for the main part of the lesson. Different variants of walking along the gym, exercises. They improve joints flexibility, are fulfilled.

The main part of the lesson includes two blocks: the first block- classical physical exercises; the second blockexercises at stable platform.

The exercises of the first block ${ }^{*}$ : in I.P. prone position at fitball, arms are put around the fitball - rolling-over forward-backward and sideways; in I.P. sitting - strumming the set rhythm with the finger; dip up with resistance overcoming, provided with the person, who organizes the lesson; one hand up, the other hand sideways; in I.P. standing- stopping the rolling ball with paretic foot; twists $360^{\circ}, 270^{\circ}, 180^{\circ}, 90^{\circ}, 45^{\circ}$. Time for the first block fulfillment 10 minutes.

The second block exercises": I.P. standing at stable platform legs shoulder-width apart - exercise "Target". It is necessary to hold the marker, which reflects pressure center position (PC) to the stable platform, in the center of the target; exercise "Audio-training simulator". It is necessary to transfer the marker, which reflects PC position on the stable platform, on the square, which constantly moves. If marker is not on the square children hear the signal of feedback in a form of a noise. It increases depending on remoteness from the square. If marker is on the square the noise stops; exercise "Pictures forming". It is necessary to form the whole picture from the parts of the picture, taking the parts of the picture with the marker; exercise "Arkanoid". It is necessary to move the platform, which reflects PC position on the stable platform and return the ball. It breaks the big figure placed at the upper part of the screen.

During the final part of the lesson relaxing exercise is fulfilled, the results of the lesson are defined.

During the exercises fulfillment at stable platform it is necessary to follow several rules:

1) Scale: 1 or 2 . The scale in computer program «StabMed» version 2.08. is the instrument. It helps to increase the sensitivity of the strain gauges. During the work with children with ICP sensitivity of the strain gauges should be no higher than 1-2 units, as stronger sensitivity makes exercises fulfillment more difficult;

2) I.P. - legs shoulder-width apart stand at a stable platform (hands long the body). The screen is placed strictly before the child at eye level. Head turns, vibrating movements with hands are forbidden, as it leads to results of satatokineseogram distortion;

3) The method of lessons organization - group, as under the influence of external stimulus (encouraging voices of coevals, other noise, attention of not known people and etc.) a child with ICP teaches to stand vibrating movements of own body and develops own coordinating abilities.

\section{Conclusion}

Thus, we offered the methodology of coordinating abilities estimation and upbringing in children with spastic diplegia and hemiparetic forms of infantile cerebral paralysis at the age of 11-12 of the Ist level of GMFCS. Coordinating abilities estimation among the tested children showed that the girls with hemiparetic form of ICP coordinating abilities are better developed in comparison with the girls with spastic diplegia and boys with both forms. After coordinating abilities estimation we would test the methodologies of coordinating abilities upbringing in the tested children, which includes the exercises at stable platform "Stabilan-01-2".

We give the exemplary list of exercises. The final list of exercises is chosen depending on the trained coordinating ability.

\section{References}

1. A.N. Nalobina, E.S. Stotskaya, I.G. Talamova, A.O. Stotskiy. Person. Sport. Medicine, 18(2), 109-118 (2018) 
2. V.A. Klendar, N.A. Gross, A.N. Korzhenevskiy, Person. Sport. Medicine. 19(3), 112-118 (2019)

3. A.S. Kuznetsov, Z.M. Kuznetsova, Russian Journal of Physical Education and Sport, 14(4), 5-9 (2019)

4. H. Chiu, L. Ada, H. Lee, Clinical Rehabilitation, 28, 1015 (2014)

5. G. Flores, J. Ordorica, Pediatric Physical Therapy, 30, 303-308 (2018)

6. J.W. Yoo, D.R. Lee, Y.J. Sim, J.H. You, C.J. Kim, Bio-Medical Materials and Engineering, 24, 3613-3618 (2014)

7. N.Yu. Kotova, A.A. Tokmakov, Pedagogical education in Russia, 1, 83-87 (2015)

8. V.K. Klimova, A.V. Posokhov, Ya.A. Strelkova, M.V. Shimokhina, Theory and Practice of Physical Culture, 1(2) (2013)

9. A.V. Poteshkin, I.G. Talamova, Adaptive Physical culture, 2 (2020)

10. A.V. Popteshkin, I.G. Talamova, Adaptive Physical culture and sport: modern state and future development, 107$111(2019)$

11. A.V. Poteshkin, I.G. Talamova, The ways of physical upbringing, sports training, health-improving and adaptive physical culture optimization, 208-210 (2019)

12. D.A. Kalmaykov, G.I. Deryabina, O.S. Terenteva, V.L. Lerner, Bulletin of Tambov University. Series: Humanitarian Sciences, 123(175), 69-80 (2018) 